\title{
ANAGRELIDE AND THE CALR MUTATION ALLELE BURDEN IN ESSENTIAL THROMBOCYTHEMIA
}

\author{
E. Atkinson', M. Bakri², A. Hayat', S.E. Langabeer ${ }^{1,}$ * \\ ${ }^{1}$ Cancer Molecular Diagnostics, St. James's Hospital, Dublin D08 E9P6, Ireland \\ ${ }^{2}$ Department of Haematology, University Hospital Galway, Galway H91 YR71, Ireland
}

The myeloproliferative neoplasm of essential thrombocythemia is characterized by a sustained peripheral blood thrombocytosis, increased numbers of morphologically abnormal megakaryocytes in the bone marrow and clinically by episodes of thrombosis or hemorrhage and the propensity to transform into myelofibrosis or acute myeloid leukemia. The main treatment goals are therefore to prevent thrombotic events and to inhibit transformation with antiplatelet and myelosuppressive drugs, respectively. The pathogenesis of essential thrombocythemia is largely defined by three types of driver mutation, namely the JAK2 V617F, CALR exon 9 and MPL exon 10 mutations. Significant improvements have been in the treatment of the related myeloproliferative neoplasm of primary myelofibrosis with JAK $1 / 2$ inhibitors, but agents such as interferon and anagrelide remain relevant therapeutic options in the modern treatment algorithm for essential thrombocythemia [1]. While hydroxyurea appears to have no effect in inducing molecular responses, reductions in the CALR mutation allele burden have been described in some essential thrombocythemia patients treated with interferon alpha and the JAK1/2 inhibitor ruxolitinib [2, 3]. Any effect of anagrelide on the mutant CALR allele burden in any essential thrombocythemia patients has not been previously described.

A 75-year old male presented with an isolated thrombocytosis of $703 \cdot 10^{9} / \mathrm{l}$. Bone marrow morphology was consistent with a diagnosis of essential thrombocythemia with clustering of increased, hyperlobulated megakaryocytes and focal reticulin deposition. The JAK2 V617F was not detected. The patient commenced aspirin $(75 \mathrm{mg}$ ) immediately and was monitored. Due to an increasing platelet count $\left(801 \cdot 10^{9} / \mathrm{l}\right)$ and white cell count $\left(20.2 \cdot 10^{9} / \mathrm{l}\right)$, hydroxyurea $(500 \mathrm{mg})$ was started 25 months post diagnosis. In the absence of a hematological response (platelet count $\left.642 \cdot 10^{9} / \mathrm{l}\right)$, anagrelide $(0.5 \mathrm{mg}$ once daily) was added increasing to $1.0 \mathrm{mg}$ and $1.5 \mathrm{mg}$ once daily over the following eight months with the patient achieving a hematological remission (platelet count $251 \cdot 10^{9} / \mathrm{I}$ and white cell count $4.2 \cdot 10^{9} / \mathrm{I}$ ). At 55 months post-diagnosis, CALR mutation analysis was performed on peripheral blood genomic DNA as previously described [4] and detected a 52 base pair deletion (type 1 mutation) in CALR exon 9 with

Submitted: May 23, 2018.

*Correspondence: E-mail: slangabeer@stjames.ie an allele burden of $54.2 \%$. Retrospective evaluation of the archival peripheral blood DNA from diagnosis revealed the same mutation present at an allele burden of $36.7 \%$. The platelet count $\left(321 \cdot 10^{9} / \mathrm{I}\right)$ and white cell count $\left(7.0 \cdot 10^{9} / \mathrm{l}\right)$ remain within normal range.

Despite anagrelide therapy, no impact on the CALR mutation allele burden was evident in this instance. While some emerging evidence suggests that driver mutation status may impact on the clinical response $[5,6]$, the above observation is broadly in line with the finding that in essential thrombocythemia, anagrelide therapy also does not impact on the JAK2 V617F allele burden $[7,8]$. While interferon and JAK $1 / 2$ inhibitors are known to exert some of their effects through disruption of immune responses, the full mechanism of action of anagrelide remains unclear with the primary effect being inhibition of megakaryocyte maturation and proplatelet formation [9]. This inability to directly target the malignant clone may reflect the inability of anagrelide to induce molecular responses in essential thrombocythemia, if indeed deep responses are required for long-term survival. This observation requires confirmation in independent patient cohorts.

\section{CONFLICTS OF INTEREST}

The authors declare no competing financial interests.

\section{REFERENCES}

1. Birgegård G. The use of anagrelide in myeloproliferative neoplasms, with focus on essential thrombocythemia. Curr Hematol Malig Rep 2016; 11: 348-55.

2. Verger E, Cassinat B, Chauveau A, et al. Clinical and molecular response to interferon-alpha therapy in essential thrombocythemia patients with CALR mutations. Blood 2015; 126: 2585-91.

3. Harrison CN, Mead AJ, Panchal A, et al. Ruxolitinib vs best available therapy for ET intolerant or resistant to hydroxycarbamide. Blood 2017; 130: 1889-97.

4. Haslam K, Langabeer SE, Molloy K, et al. Assessment of CALR mutations in myelofibrosis patients, post-allogeneic stem cell transplantation. Br J Haematol 2014; 166: 800-2.

5. Iurlo A, Cattaneo D, Orofino N, et al. Anagrelide and mutational status in essential thrombocythemia. BioDrugs 2016; 30: 219-23.

6. Mela Osorio MJ, Ferrari L, Goette NP, et al. Long-term follow-up of essential thrombocythemia patients treated with anagrelide: subgroup analysis according to JAK2/CALR/MPL mutational status. Eur J Haematol 2016; 96: 435-42.

7. Larsen TS, Pallisgaard N, de Stricker K, et al. Limited efficacy of hydroxyurea in lowering the JAK2 V617F allele burden. Hematology 2009; 14: 11-5. 
8. Cascavilla N, De Stefano V, Pane F, et al. Impact of JAK2 (V617F) mutation status in treatment response to anagrelide in essential thrombocythemia: an observational, hypothesis-generating study. Drug Des Devel Ther 2015; 9: $2687-94$
9. Espasandin YR, Glembotsky AC, Grodzielski M, et al. Anagrelide platelet-lowering effect is due to inhibition of both megakaryocyte maturation and proplatelet formation: insight into potential mechanisms. J Thromb Haemost 2015; 13: 631-42. 